\title{
Status and perspectives of the radio detection of high- energy cosmic rays
}

\author{
Jörg R. Hörandel ${ }^{1,2,3, *}$ \\ ${ }^{1}$ Radboud Universiteit, Department of Astrophysics/IMAPP, P.O. Box 9010, 6500 GL Nijmegen, The \\ Netherlands \\ ${ }^{2}$ Nikhef, Science Park 105, 1098 XG Amsterdam, The Netherlands \\ ${ }^{3}$ Vrije Universiteit Brussel, Department of Physics and Astronomy, B-1050 Brussels, Belgium
}

\begin{abstract}
Status and perspectives of the radio detection of high-energy cosmic rays are discussed with emphasis on recent developments at the LOFAR radio telescope and the Pierre Auger Observatory.
\end{abstract}

\section{Introduction}

High-energy cosmic rays impinging on the atmosphere of the Earth initiate cascades of secondary particles, the extensive air showers. Many secondary particles are electrons and positrons. They emit electromagnetic radiation with frequencies of tens of $\mathrm{MHz}$ during the shower development. The bulk of the radiation originates from the transverse separation of charges in the shower due to interactions with the Earth's magnetic field: the geomagnetic effect [1]. One expects the radiation intensity being proportional to $v \times B$, where $v$ denotes the direction of the particles in the shower (shower axis) and $B$ the Earth magnetic field. The geomagnetic radiation is linearly polarized.

A second, sub-dominant component arises through the longitudinal separation of charges in the shower (Askaryan effect [2]). This component is radially polarized. The direction of the electric field within the shower is measured with high resolution with LOFAR [3]. This allows one to quantitatively evaluate the contribution of the two emission processes to the overall shower emission. The results yield a dependence on the zenith angle and the distance to the shower axis for the ratio of the two radiation components. Values for the ratio range from about $5 \%$ to $20 \%$, with an average contribution of the Askaryan effect of about $15 \%$. A similar value has been obtained through polarization measurements with AERA [4].

Interference between the two radiation components with their different polarization behavior leads to a non-rotational symmetric distribution of the radiation intensity on the ground, as illustrated in Fig. 1 (left). This implies a non-trivial behavior of the lateral density distribution of the radio emission. Using the dense LOFAR core $[8,9]$ it became obvious that the lateral density distribution is not rotationally symmetric and a simple description as e.g. proposed in [10] is not sufficient. Instead now, more complex, two-dimensional functions are being used to describe the azimuthal dependence of the radiation strength $[5,8]$. As an example, a parameterization introduced in [8] is depicted in Fig. 1 (right). The colors represent different intervals in azimuthal angles. The non-rotational symmetry is clearly visible.

\footnotetext{
*e-mail: j.horandel@astro.ru.nl
} 

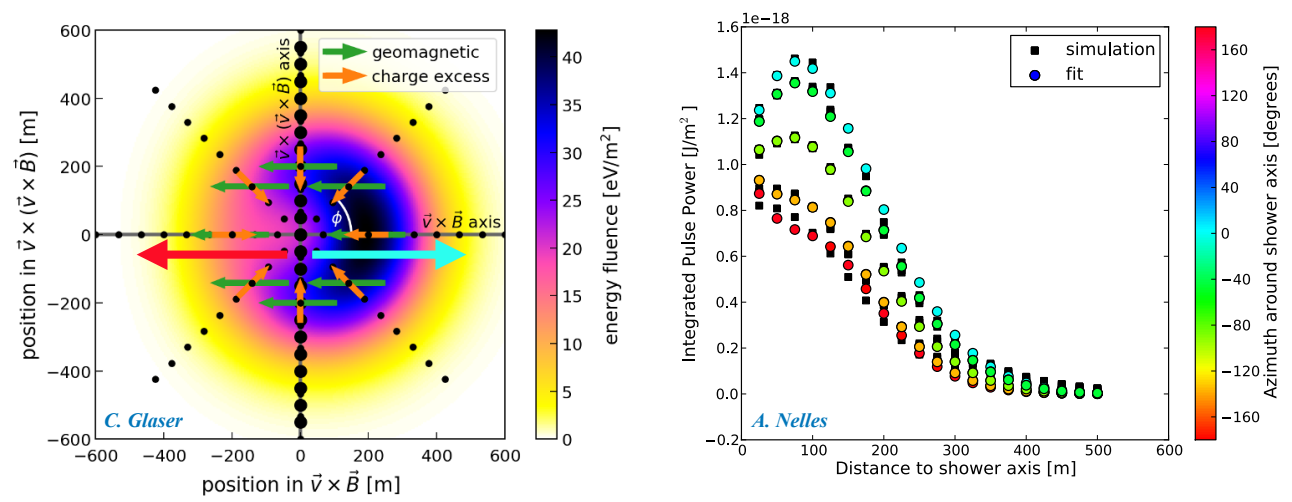

Figure 1. Left: Distribution of the energy fluence (in the $30-80 \mathrm{MHz}$ band) of an air shower with $60^{\circ}$ zenith angle [5]. Superimposed is the polarization direction of the geomagnetic and charge-excess emission processes at different positions in form of arrows. Simulated with CORSIKA/CoREAS [6, 7]. Right: Lateral distribution of the radiation power on the ground for bins in azimuthal angle, following a parameterization described in [8].

\section{LOFAR and AERA}

We focus here on the description of recent results obtained by the LOFAR radio telescope and the Auger Engineering Radio Array (AERA) at the Pierre Auger observatory.

LOFAR $[11,12]$ is a distributed radio telescope. Its antennas are distributed over northern Europe with the densest concentration in the north of the Netherlands. The antennas of LOFAR are grouped into stations. A station consist of 96 low-band antennas $(10-90 \mathrm{MHz})$ and 48 high-band antennas $(110-240 \mathrm{MHz})$. The 23 stations within the $\sim 5 \mathrm{~km}^{2}$ core are distributed in an irregular pattern that maximizes uv-coverage, or spatial frequencies for standard interferometric observations. At the center of the LOFAR core six stations are located in a roughly $320 \mathrm{~m}$ diameter area, called the Superterp, providing both, the shortest baselines for interferometric observations and the densest population of antennas, ideal for cosmic-ray observations. An array of scintillation detectors installed on the Superterp complements the set-up $[13,14]$. The particle detector array is used to measure the properties of air showers and to issue a trigger signal to read out the radio antennas.

The AERA project $[15,16]$ comprises more than 150 antennas installed on an area covering about $17 \mathrm{~km}^{2}$ inside the Auger observatory in the field of view of the High Elevation Telescopes (HEAT). HEAT are fluorescence light detectors aimed to measure air showers with energies above $10^{17} \mathrm{eV}$. The radio antennas are installed on sub-grids with different spacings, ranging from $144 \mathrm{~m}$ to $375 \mathrm{~m}$ in order to compensate for the change of the size of the radio footprint as a function of cosmic-ray energy. Two types of antennas are installed [17]: 24 stations are equipped with two logarithmic-periodic dipole antennas, oriented in magnetic North-South and East-West directions. The signals are amplified and filtered before they are introduced into a filter-amplifier and digitization chain. There, they are band pass filtered between 30 and $80 \mathrm{MHz}$ and digitized. The remaining antennas are an active bowtie antenna, called "butterfly antennas". This antenna is highly sensitive towards the ground which enhances the antenna gain. All radio detector stations operate autonomously employing solar power systems.

Both installations are calibrated in situ with high precision, both for the signal strength $[18,19]$ and the signal arrival time $[20,21]$. 


\section{Properties of cosmic rays}

The ultimate goal of the radio measurements of air showers is to derive the properties of the incoming cosmic rays, namely their arrival direction, energy and particle type (nuclear mass).

The direction of the cosmic ray is obtained through a precise measurement of the arrival time of the wavefront at the individual antennas of an array. LOFAR, with its high antenna density was valuable to clearly understand the precise shape of the radio wavefront in air showers [22] and it could be shown that a hyperboloid is an appropriate analytical description. This confirms earlier work by LOPES [23]. From measurements of the arrival time of the shower front the direction of the cosmic rays can be determined with a resolution of better than $1^{\circ}$.

The energy of the air shower is derived from the measurement of the radio energy density on the ground [24, 25]. A function [8] is fitted to the measured values in order to calculate the integral of the total energy in the frequency band $30-80 \mathrm{MHz}$ transported in an air shower to the ground. This quantity is proportional to the shower energy. For a set of high-quality showers an energy resolution of better than $25 \%$ has been achieved [24, 25].

At LOFAR, using results from simulations and a comparison to measurements from a particle detector array an energy resolution around 30\% is obtained [9, 26].

A cosmic ray with an energy of $1 \mathrm{EeV}$ delivers about $15.8 \mathrm{MeV}$ of energy to the ground in the frequency range from 30 to $80 \mathrm{MHz}[24,25]$. The radio emission measured on the ground $E_{30-80 \mathrm{MHz}}$ can be used to establish an absolute calibration of the energy scale $E_{\mathrm{CR}}$, using the universal formula

$$
\begin{aligned}
E_{30-80 \mathrm{MHz}}=[15.8 & \pm 0.7 \text { (stat) } \pm 6.7 \text { (syst) }] \mathrm{MeV} \\
& \times\left(\sin \alpha \frac{E_{\mathrm{CR}}}{10^{18} \mathrm{eV}} \frac{B_{\text {Earth }}}{0.24 \mathrm{G}}\right)^{2},
\end{aligned}
$$

knowing the magnetic field $B_{\text {Earth }}$ at the location of the detector and the angle $\alpha$ between the direction of the Earth magnetic field and the direction of the air shower.

Experimentally, the most challenging subject is the determination of the particle type or its nuclear mass. The basic observable in air shower physics is the depth of the shower maximum $X_{\max }$, being proportional to the nuclear mass $A$ of the cosmic ray $X_{\max } \propto \ln A$. The radio signals are in principle emitted on the Čerenkov cone. Thus, the size of the footprint of the radio emission on the ground scales well with the distance from the emission region (basically $X_{\max }$ ) to the antennas (on the ground) [9]. Knowing the vertical profile of the atmosphere, the depth of the shower maximum $X_{\max }$ can be derived. For LOFAR a high-precision method has been developed to measure $X_{\max }[27,28]$. For each measured cosmic ray, dedicated simulations are conducted, taking into account the measured energy and direction of the cosmic ray. The predicted signals in the particle and radio detectors are compared to the measured values on a statistical basis and a best fit value is obtained for $X_{\max }$. A resolution fo $X_{\max }$ of better than $20 \mathrm{~g} / \mathrm{cm}^{2}$ has been obtained.

This method is also applied to AERA data. The obtained $X_{\max }$ values from the radio measurements have been compared to $X_{\max }$ obseravtions from the fluorescence light telescopes. In the first analyses a resolution of the order of $40 \mathrm{~g} / \mathrm{cm}^{2}$ has been achieved. The resolution depends on the number of antennas participating in a measurement and thus, on the quality selection cuts applied [29].

Combining the various efforts around the world one can state that the radio detection technique is now mature and the properties of cosmic rays are now being measured on a regular basis with such devices with state-of-the-art accuracies of the order of $0.1^{\circ}$ to $0.5^{\circ}$ for the arrival direction, $20 \%$ to $30 \%$ for the energy, and 20 to $40 \mathrm{~g} / \mathrm{cm}^{2}$ for the depth of the shower maximum (used to study the cosmic-ray mass - particle type), with the accuracies mainly depending on the antenna density of the respective installation. 

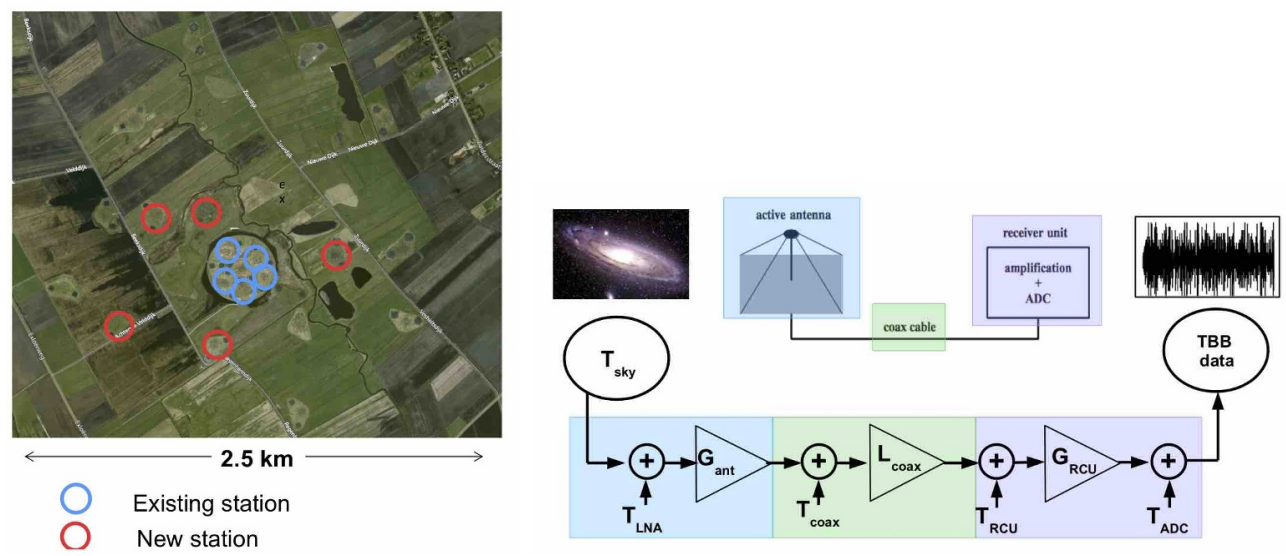

Figure 2. Left: Satellite image of the LOFAR dense core, illustrating the extension of the scintillator array. Blue circles indicate existing stations with particle detectors. In addition, particle detectors will be installed at the stations indicated with red circles. Right: LOFAR signal chain, illustrating the various steps from the observed signal on the sky to recorded ADC values, stored in ring buffers (transient buffer boards - TBB) [30].

\section{Ongoing work and perspectives for the future}

\subsection{LOFAR}

At present the particle detector array at the LOFAR observatory is being extended. Five LOFAR stations around the Superterp are being equipped with scintillator stations, as illustrated in Fig. 2 (left). At each station four scintillator detectors, $1 \mathrm{~m}^{2}$ each, identical to the ones described in [13] will be installed. This will increase the effective area of the LORA particle detector array. At energies between $10^{16}$ and $10^{18} \mathrm{eV} \mathrm{a} \approx 45 \%$ increase of the number of air showers with a radio signal is expected [31].

At present also the calibration procedure for LOFAR is being improved [30], further reducing the systematic uncertainties with respect to previous work [18]. Radio emission from the Galactic center is used as a reference source. The complete signal chain, end to end, from the signal on the sky to the recorded ADC traces is evaluated, as illustrated in Fig. 2 (right). This method requires knowing the contributions of electronic noise to the system, and the original Galactic calibration is limited by the systematic uncertainty of the electronic noise. This is being improved through the ongoing efforts by modeling each step in the signal chain in order to understand the electronic noise introduced into the system. Using this method, a new absolute and frequency dependent calibration has been derived with a maximum of $14 \%$ systematic uncertainty for frequencies below $77 \mathrm{MHz}$. A comparison of the logarithmic slopes of calibrated, measured power spectra and simulated power spectra shows promising agreement.

\subsection{The radio upgrade of the Pierre Auger observatory}

At present, the Pierre Auger collaboration is working on an upgrade of the Observatory [33]. The physics case of the upgrade is outlined in [34]. The key science questions to be addressed are: What are the sources and acceleration mechanisms of ultra-high-energy cosmic rays (UHECRs)? Do we understand particle acceleration and physics at energies well beyond the 

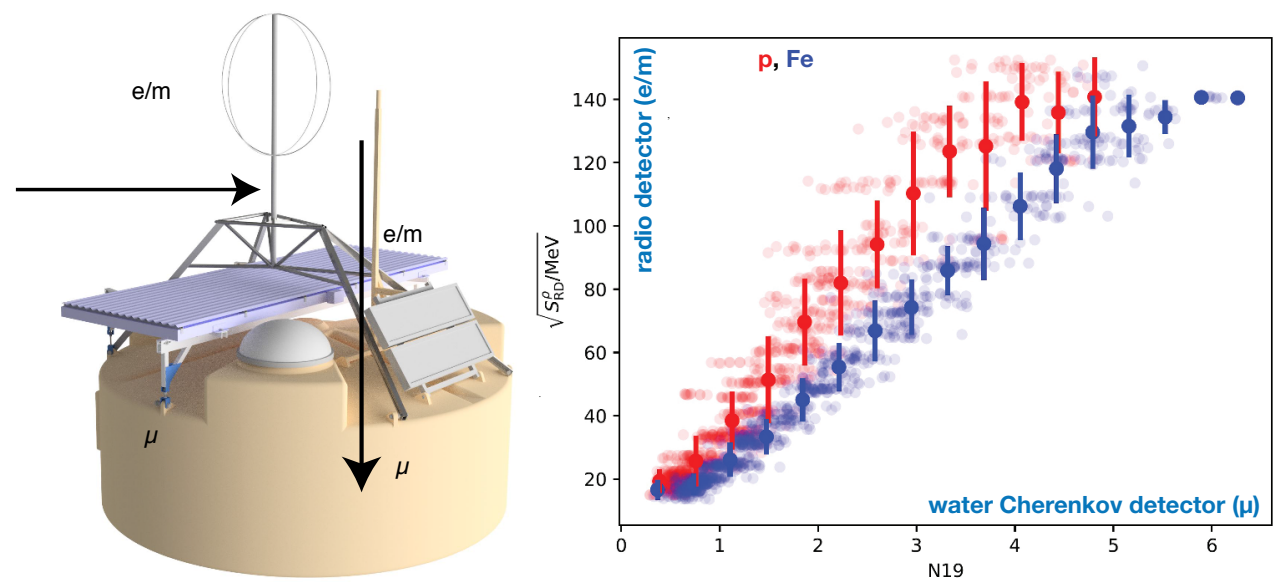

Figure 3. Left: Conceptional illustration of an upgraded station of the Surface Detector array of the Pierre Auger observatory, comprised of (from bottom to top) a water-Čerenkov detector, a layer of scintillators, and a radio antenna [32]. The arrows indicate the detection principles for vertical and horizontal air showers. In practice, the electromagnetic and muonic components are obtained by unfolding the signals from the Surface Scintillator Detector and the water-Čerenkov detector. Right: Electromagnetic energy in a shower as measured by the radio detector as a function of the muon content in a shower as measured by the water-Čerenkov detector [32]. Preliminary results from simulations are shown, see main text for more details.

LHC (Large Hadron Collider) scale? What is the fraction of protons, photons, and neutrinos in cosmic rays at the highest energies?

It is planned to install a radio antenna on each of the 1661 stations of the Surface Detector array of the observatory, forming a $3000 \mathrm{~km}^{2}$ radio array, the largest radio array for cosmic-ray detection in the world [32,35]. An artists impression of the planned set-up is given in Fig. 3 (left). It shows (from bottom to top) the water-Čerenkov detector with a layer of scintillators on top (Surface Scintillator Detector) and a radio antenna. With the combination of water-Čerenkov detector and Surface Scintillator Detector the electron-to-muon ratio $(\mathrm{e} / \mu)$ is measured for vertical showers. In a similar way the combination of water-Čerenkov detector and Radio Detector will be used to measure the $\mathrm{e} / \mu$ ratio for air showers with large zenith angles, in the following called horizontal air showers. In turn, the e $/ \mu$ ratio will be used to derive the particle type of the incoming cosmic ray up to the highest energies. This is the main goal of the Auger upgrade, to measure the particle type of each incoming cosmic ray. Thus, the radio upgrade will increase the aperture of the observatory for mass-sensitive investigations, allowing to do e $/ \mu$ separation for showers with a broad zenith angle range, from zenith with the Surface Scintillator Detector to the horizon with the Radio Detector.

There is an important difference related to the mass measurement of cosmic rays with AERA (for vertical showers) and the radio upgrade (for horizontal air showers). For vertical showers we use a geometrical method, correlating the size of the footprint on the ground to the distance from the observer to the shower maximum [15, 26, 36]. From this quantity, the depth of the shower maximum $X_{\max }$ is derived, which in turn is dependent on the logarithm of the number of nucleons (nuclear mass) $A$ of the incoming cosmic ray $X_{\max } \propto \ln A$ [15]. For horizontal showers we aim to apply a different method: We will use a combination of radio antennas and the water-Čerenkov detectors to measure the e $/ \mu$ ratio in air showers to determine $\ln A$. 
In a first analysis the physics potential of the radio upgrade has been studied using CORSIKA[6]/CoREAS[7] simulations. 192 showers have been simulated, induced by half protons, have iron nuclei, with energies from 4 to $40 \mathrm{EeV}$ and zenith angles between $60^{\circ}$ to $80^{\circ}$. A full water-Čerenkov detector detector simulation and reconstruction has been included in the analysis, but only a simplified treatment of the radio signals was available. The normalized radiation energy has been calculated as described in [37]. The radiation energy has been "smeared out" in order to mimic reconstruction uncertainties. Preliminary results are illustrated in Fig. 3 (right). The size of the e/m component (as measured with the Radio Detector) is plotted as a function of the muon number (as obtained with the water-Čerenkov detector). A clear separation between showers induced by protons and iron nuclei is visible. Please note: the flattening at the top-right is due to a technical flaw in the simulations, only showers up to a certain (too low) energy have been simulated.

The concept to measure horizontal air showers with an antenna array with $\mathrm{km}$-scale spacing has been verified with the existing AERA at the Auger observatory [38, 39]. The size of the radio footprint of an air shower has been measured. The distance from the shower axis to the furthest away radio detector (in the shower plane, perpendicular to the shower axis) has been evaluated. For horizontal air showers (i.e. large zenith angles) the footprints reach sizes exceeding tens of $\mathrm{km}^{2}$ and dozens of stations will have a signal above threshold. This is a very important result since it confirms experimentally that the radio emission from horizontal air showers can be measured with radio antennas on the standard Auger $1500 \mathrm{~m}$ grid.

\section{References}

[1] F. Kahn, I. Lerche, Royal Society of London Proceedings Series A 289, 206 (1966)

[2] G. Askaryan, Journal of the Physical Society of Japan Supplement 17, C257 (1962)

[3] P. Schellart, et al., JCAP 1410, 014 (2014)

[4] A. Aab et al. (Pierre Auger Collaboration), Phys.Rev. D89, 052002 (2014)

[5] C. Glaser, S. de Jong, M. Erdmann, J.R. Hörandel, Astropart. Phys. 104, 64 (2019)

[6] D. Heck et al., Corsika: A monte carlo code to simulate extensive air showers, Report FZKA 6019, Forschungszentrum Karlsruhe (1998)

[7] T. Huege, M. Ludwig, C.W. James, ARENA 2012, AIP Conf. Proc. 1535 pp. 128-132 (2013)

[8] A. Nelles, et al., Astropart.Phys. 60, 13 (2015)

[9] A. Nelles, et al., JCAP 1505, 018 (2015)

[10] H. Allan, Progress in Elementary Particles and Cosmic Ray Physics (J.G. Wilson and S.G. Wouthuysen eds., North Holland, 1971), p. 169

[11] M. van Haarlem, et al., Astronomy \& Astrophysics 556, A2 (2013)

[12] P. Schellart, et al., Astron.Astrophys. 560, A98 (2013)

[13] S. Thoudam, S. Buitink, A. Corstanje, J. Enriquez, H. Falcke et al., Nucl.Instrum.Meth. A767, 339 (2014)

[14] S. Thoudam et al., Astropart. Phys. 73, 34 (2016)

[15] J. Schulz, Pierre Auger Collaboration, Proceedings 34th International Cosmic Ray Conference, Den Haag p. PoS(ICRC2015)615 (2015)

[16] P. Abreu et al. (Pierre Auger Collaboration), Nucl.Instrum.Meth. A635, 92 (2011)

[17] P. Abreu et al. (Pierre Auger Collaboration), JINST 7, P10011 (2012)

[18] A. Nelles et al., JINST 10, P11005 (2015)

[19] A. Aab et al. (Pierre Auger), JINST 12, T10005 (2017)

[20] A. Corstanje et al., Astron. Astrophys. 590, A41 (2016) 
[21] A. Aab et al. (Pierre Auger), JINST 11, P01018 (2016)

[22] A. Corstanje, et al., Astropart.Phys. 61, 22 (2015)

[23] W. Apel, J. Arteaga-Velázquez, L. Bähren, K. Bekk, M. Bertaina et al., JCAP 1409, 025 (2014), 1404.3283

[24] A. Aab et al. (Pierre Auger), Phys. Rev. Lett. 116, 241101 (2016)

[25] A. Aab et al. (Pierre Auger), Phys. Rev. D93, 122005 (2016)

[26] S. Buitink, et al., Phys.Rev. D90, 082003 (2014)

[27] S. Buitink et al., Proceedings 34th International Cosmic Ray Conference, Den Haag p. PoS(ICRC2015)368 (2015)

[28] S. Buitink et al., Nature 531, 70 (2016)

[29] F. Canfora et al., Proceedings ARENA 2018 (2018)

[30] K. Mulrey et al., Proceedings ARENA 2018 (2018)

[31] S. Buitink et al., Proceedings ARENA 2018 (2018)

[32] J. Hörandel, Proceedings ARENA 2018 (2018)

[33] A. Castellina et al., Proceedings UHECR symposium, Paris (2018)

[34] A. Aab et al. (Pierre Auger), arXiv p. 1604.03637 (2016)

[35] J. Hörandel, Proceedings UHECR symposium, Paris (2018)

[36] T. Huege et al., Proceedings UHECR symposium, Paris (2018)

[37] C. Glaser, M. Erdmann, J.R. Hörandel, T. Huege, J. Schulz, JCAP 1609, 024 (2016)

[38] A. Aab et al. (Pierre Auger), JCAP 1810, 026 (2018)

[39] M. Gottowik et al., Proceedings ARENA 2018 (2018) 